\title{
An Efficient Algorithm for Fast Computation of Pseudo-Zernike Moments
}

\author{
Chong, Chee-Way \\ Fac. of Engg \& Tech., \\ Multimedia University, \\ Jalan Air Keroh Lama, \\ 75450 Melaka, Malaysia. \\ (Email:cwayc@pc.jaring.my)
}

\author{
R.Mukundan \\ Fac. of Information Science \\ \& Technology, \\ Multimedia University, \\ Melaka, Malaysia. \\ (Email:mukund@mmu.edu.my)
}

\author{
P.Raveendran \\ Department of Electrical \\ Engineering, \\ University of Malaya \\ Kuala Lumpur, Malaysia. \\ (Email:ravee@fk.um.edu.my)
}

\begin{abstract}
Pseudo-Zernike moments have better feature representation capabilities and are more robust to image noise than the conventional Zernike moments. However, pseudo-Zernike moments have not been extensively used as feature descriptors due to the computational complexity of the pseudo-Zernike radial polynomials. This paper discusses the drawbacks of the existing methods, and proposes an efficient recursive algorithm to compute the pseudo-Zernike moments. The algorithm consists of a two-stage recurrence relation for radial polynomials and coefficients of the polynomials, which are specifically derived for fast computation of pseudo-Zernike moments. The performance of the algorithm is experimentally examined using both binary and grayscale images, and it shows that the computational speed of pseudo-Zernike moments has been substantially improved over the present methods.
\end{abstract}

\section{Introduction}

Pseudo-Zernike moments are used in several pattern recognition applications [1-3] as feature descriptors of the image shape, and have proven to be superior to other moment functions such as Zernike moments in terms of their feature representation capabilities and robustness in the presence of image quantization error and noise [4]. Pseudo-Zernike moments offer more feature vectors than Zernike moments since pseudo-Zernike polynomial contains $(p+1)^{2}$ linearly independent polynomials of order $\leq p$, whereas Zernike polynomial contains only $\frac{1}{2}(p+1)(p+2)$ linearly independent polynomials due to condition of $p-q=$ even [4]. Teh et al. have also proven that pseudo-Zernike moments are less sensitive to image noise than are the conventional Zernike moments in [4].
Further, their orthogonality property of the polynomials helps in achieving a near zero value of redundancy measure in a set of moment functions, so that moments of different orders correspond to independent characteristics of the image [4-7].

Pseudo-Zernike moments have not been effectively used in image processing applications because their computation with present methods is however more complex and lengthy compared to regular moments, Legendre moments and even Zernike moments. In addition, most researchers focused only on algorithms for fast computation of Zernike moments and in most cases, they cannot be directly applied to the computation of pseudo-Zernike moments [8,12-14]. The present method of direct computation of radial polynomials is merely sufficient to obtain a single pseudo-Zernike moment of order $p$ in a single run. For complete computation of order $p$, it has to go through the same loop repeatedly. This approach takes much longer time since it involves the factorial iterations for each radial polynomial, and may lead to numerical instabilities as the order $p$ increases.

Meanwhile, Mukundan et al. have proposed a fast algorithm that employs the contour integration method and square-to-circular method to compute Zernike moments [8-11]. With minor modifications, this technique can be utilized to compute pseudo-Zernike moments. However, the former method is applicable only to binary images and it requires off-line analysis to extract the image boundary points, whereas the latter technique will differ from the true moments of the rectangular image. Belkasim too has introduced an algorithm for fast computation of Zernike moments based on the series angular and radial expansion of the radial polynomials [13]. Even though this method can also be adapted to compute the pseudo-Zernike moments, it will not significantly improve the evaluation speed of pseudoZernike moments due to the factorial functions of the coefficients in radial polynomials. 
In this contribution, an efficient two-stage recursive algorithm is introduced to reduce the complex computation involved in radial polynomials. The algorithm comprises of a couple of recurrence relations for radial polynomials and coefficients of the polynomials, which are specifically derived for fast computation of pseudo-Zernike moments. The proposed recurrence relation for coefficients of radial polynomials, denoted hereafter as coefficient method is free from any factorial functions, which contribute most of the computation time of pseudo-Zernike moments. Further, the entire set of pseudo-Zernike moments of order $p$ and $q$-repetition can be obtained concurrently in a single run of the algorithm. It is suitable to be applied in parallel processing environment since each order $p$ can be scheduled to different processing elements.

However, the amount of computation time is still substantial for coefficient method if it is carried out sequentially to calculate the entire set of pseudo-Zernike moments using a single processing element. There is only one set of pseudo-Zernike moments of order $p$ can be computed at one time. The evaluation of remaining pseudo-Zernike moments of order $p-1, p-2, p-3$ etc is suspending, though they can be computed exclusively from each other. Apart from this, this method still needs to calculate each and every coefficient and the power series of radius involved in radial polynomial though all the terms can be derived at the same loop.

In order to resolve the above problem, a recurrence relation for radial polynomials, denoted hereafter as recursive method is proposed to enable the higher order radial polynomials to be derived from the lower order radial polynomials without computing the details of each radial polynomial. Since the recurrence relation is only limited in computing the radial polynomials of order $(p-q$ $\geq 2$ ), the coefficient method is used to calculate the remaining radial polynomials of order $(p-q<2)$. The integration of the recurrence relations from recursive method and coefficient method, denoted as two-stage recursive method is optimizied to simplify the evaluation of radial polynomials and release the computation of their respective coefficients from involvement of factorial terms. It can be tuned for serial, parallel or serial-parallel processing architecture to achieve optimal computational speed of pseudo-Zernike moments.

In the experimental study, five methods are examined to determine their respective computation speed to calculate the pseudo-Zernike moments using binary and grayscale images under a single processing unit. They consist of coefficient method, recursive method along with direct method for order $(p-q<2)$, two-stage recursive method, direct method and modified Belkasim's method. The results showed that the two-stage recursive method followed by the recursive method took the shortest time to compute the entire set of pseudo-Zernike moments for both binary and grayscale images using single processing unit. It also showed that the coefficient method is more efficient than two-stage recursive method and recursive method in computing specific order of pseudo-Zernike moments.

\section{Pseudo-Zernike Moments}

The kernel of pseudo-Zernike moments is the set of orthogonal pseudo-Zernike polynomials defined over the polar coordinates inside a unit circle. The twodimensional pseudo-Zernike moments of order $p$ with repetition $q$ of an image intensity function $f(r, \theta)$ are defined as [15]:

$Z_{p q}=\frac{p+1}{\pi} \int_{0}^{1} \int_{-\pi}^{\pi} R_{p q}(r) e^{-j q \theta} f(r, \theta) r d r d \theta$

where

$$
\begin{gathered}
r=\sqrt{x^{2}+y^{2}}, \quad \theta=\tan ^{-1}\left(\frac{y}{x}\right), \quad-1<x, y<1, \\
R_{p q}(r)=\sum_{s=0}^{p-|q|}(-1)^{s} \frac{(2 p+1-s) !}{s !(p+|q|+1-s) !(p-|q|-s) !} \cdot r^{p-s}
\end{gathered}
$$

and

$$
0 \leq|q| \leq p, p \geq 0
$$

Since it is easier to work with real functions, $Z_{p q}$ is often split into its real and imaginary parts, $\mathrm{Z}_{p q}^{c}$ and $\mathrm{Z}_{p q}^{s}$ as given below:

$$
\begin{aligned}
& Z_{p q}^{c}=\frac{2(p+1)}{\pi} \int_{0}^{1} \int_{-\pi}^{\pi} R_{p q}(r) \cos (q \theta) f(r, \theta) r d r d \theta \\
& Z_{p q}^{s}=\frac{2(p+1)}{\pi} \int_{0}^{1} \int_{-\pi}^{\pi} R_{p q}(r) \sin (q \theta) f(r, \theta) r d r d \theta
\end{aligned}
$$

where $p \geq 0$ and $q>0$

\section{Fast Computation of Pseudo-Zernike Moments}

The fast computation of pseudo-Zernike moments using the existing methods is mainly resisted by factorial terms involved in the coefficient of radial polynomial, and the need to calculate each and every coefficient and the power series of radius involved in radial polynomials. In view of these limitations, we address a two-stage recursive algorithm where a couple of recurrence relations are specifically derived for pseudo-Zernike radial polynomials and their coefficients for fast compuation of pseudo-Zernike moments. The first stage recurrence 
relation, known as recursive method, associates the $R_{p q}$ with $R_{(p-1) q}$ and $R_{(p-2) q}$ which is given as follows:

$$
R_{p q}(r)=\left(L_{1} r^{2}+L_{2}\right) R_{(p-1) q}(r)+L_{3} R_{(p-2) q}(r)
$$

where the coefficients $L_{1}, L_{2}$ and $L_{3}$ are given by

$$
\begin{aligned}
L_{1}= & \frac{(2 p+1)(2 p)}{(p+q+1)(p-q)} ; \\
L_{2}= & -2 p+\frac{(p+q)(p-q-1)}{(2 p-1)} L_{1} ; \\
L_{3}= & (2 p-1)(p-1)-\frac{(p+q-1)(p-q-2)}{2} L_{1}+ \\
& 2(p-1) L_{2} ;
\end{aligned}
$$

It is clearly seen that the recurrence relation of radial polynomials enables the higher order radial polynomials to be derived from the lower order radial polynomials without computing the details of each radial polynomial. According to the recurrence relation, $R_{p q}$ relies on $R_{p-1 q}$ and $R_{p-2, q}$, and these two terms also count on the next $R_{p-3, q}$ and $R_{p 4, q}$, which have not been previously found. In view of this inter-dependent relationship, the $R_{p q}, R_{p-1 q}, R_{p-2, q}$ etc cannot be evaluated independently and parallelly. Further, it should be noted that the above relation provides a recursive scheme over the index $p$ and $q$ except for order $p-q<2$. As a result, radial polynomials of orders $p-q<2$ must be explicitly obtained using direct method. This inherent disability to compute for order $p-q<2$ extends the computation time for the whole process due to involvement of factorial iterations from direct method.

In order to avoid the involvement of factorial function contained in direct method, we propose another recurrence relation, known as coefficient method to compute the pseudo-Zernike radial polyniamls of order $p-$ $q<2$. If we apply the transformation of the index $s \leftarrow(p-$ $2 s$ ), then equation (2) can be rewritten in the form:

$$
R_{p q}(r)=\sum_{s=q}^{p} B_{p q s} r^{s}
$$

where

$$
B_{p q s}=\frac{(-1)^{p-s}(p+s+1) !}{(p-s) !(s+q+1) !(s-q) !}
$$

From the above expression, the following recurrence relations in the coefficients $B_{p q s}$ are derived:

$$
\begin{aligned}
& B_{p p p}=1 \\
& B_{p(q-1) s}=B_{p q s} \frac{(s+q+1)}{(s-q+1)} \\
& B_{p q(s-1)}=-B_{p q s} \frac{(s+q+1)(s-q)}{(p+s+1)(p-s+1)}
\end{aligned}
$$

The fast computation of $p^{\text {th }}$ order pseudo-Zernike radial polynomials can be obtained by calculating the coefficient of $r^{s}$ i.e. $B_{p q s}$, which is schematically tabulated in Table 1. According to Table 1, the computation flow starts from $B_{p p p}(=1)$ and proceeds to obtain $B_{p, p-1, p}$ and $B_{p, p-2, p}$ using equation (12). With these initial values, another set of the coefficients $B_{p, p-1, p-1}, B_{p, p-2, p-1}$ and $B_{p, p-}$ $2, p-2$, are obtained using equation (13). This process will keep on generating the coefficients of $p^{\text {th }}$ order radial polynomials until the conditions of equation (9) are met.

The coefficient method is free from any factorial functions, which contribute most of the computation time of pseudo-Zernike moments. Further, the entire set of pseudo-Zernike moments of order $p$ and $q$-repetition can be obtained concurrently in a single run of the algorithm. The coefficient method is also able to compute any arbitrary order $p$ of pseudo-Zernike moments independently without any assistance from the previous order $p-1, p-2$ etc. Thus, it is utilized to resolve the limitation of recursive method by introducing the following formula to compute orders of $p-q<2$ of pseudoZernike radial polynomials:

$$
R_{p, p-1}(r)=(2 p+1) R_{p p}(r)+(2 p) R_{p-1, p-1}(r)
$$

The integration of recursive method and coefficient method is denoted as two-stage recursive method. To illustrate the algorithm, the whole process of computing the radial polynomials is described using an example for polynomials of order 5 as shown in Figure 2. Using the recurrence relation of coefficient method derived in equation (11), the entire set of radial polynomials with order $p=q$ namely $R_{55}, R_{44}, R_{33}, R_{22}, R_{11}$ and $R_{00}$ can be easily obtained using $R_{p p}=r^{p}$ (15) as shown in the layer of dotted text box. With these initial values, the radial polynomials $R_{p, p-1}$ namely $R_{54}, R_{43}, R_{32}, R_{21}$, and $R_{10}$ can be subsequently calculated using equations (14) as illustrated using shaded text box in Figure 2. Next, with these initial values $R_{p p}$ and $R_{p p-1}$, the remaining radial polynomials of order $p-q \geq 2$ can be evaluated using equation (5) from recursive method.

With the equations of (5), (14) and (15), the two-stage recursive method excludes the participation of factorial terms for pseudo-Zernike moments of order $p-q<2$, and simplifies the evaluation of radial polynomials without taking into account of details of radial polynomials of order $p-q \geq 2$. It has fully resolved the limitations in recursive method as well as coefficient method, and it can be tuned for serial, parallel or serial-parallel architecture to achieve optimal computational speed of pseudoZernike moments. 


\section{Experimental Study}

A 50x50 binary and a grayscale image of Chinese characters shown in respective Figures 1(a) and (b) are used to examine the computation speed of the existing algorithms as well as the proposed algorithms. They comprise of direct method, modified Belkasim's method, coefficient method, recursive method and two-stage recursive method. They are used to compute 48 orders of pseudo-Zernike moments and the CPU time (in seconds) ellapsed for each order of moment is then collected under a single processing element environment. Table 2 and 3 tabulates the selected results for order 24,36 and 48 , which show that as the order increases, the two-stage recursive method took the shortest time to compute the pseudo-Zernike moments using binary image as well as grayscale image.

In order to analyze the time taken for each order of pseudo-Zernike moments, the CPU ellapsed time for $48^{\text {th }}$ order moment using coefficient method, recursive method and two-stage recursive method is tabulated in Table 4. It is calculated from the computation time difference between order $p$ and $p+1$ of pseudo-Zernike moments for binary and grayscale images. It can be seen that the maximum time taken by coefficient method to completely compute the $48^{\text {th }}$ order of pseudo-Zernike moments is merely $0.3 \mathrm{~s}$ and $1.9 \mathrm{~s}$ for the respective binary and grayscale images. This is a tremendous improvement over the corresponding $1.2 \mathrm{~s}$ and $7.0 \mathrm{~s}$ of two-stage recursive method, and $1.4 \mathrm{~s}$ and $8.2 \mathrm{~s}$ of recursive method.

\section{Conclusion}

In this paper, we discussed the limitations of existing methods, and proposed an efficient two-stage recursive method to compute pseudo-Zernike moments. The algorithm comprises of two recurrence relations that are specifically derived for radial polynomials and coefficients of the polynomials for fast computation of pseudo-Zernike moments. The performance of each method has been experimentally analyzed by taking their respective CPU ellapsed time in calculating the pseudoZernike moments for binary and gray level images. The results proved that the proposed two-stage recursive method took the shortest time in computing the entire set of pseudo-Zernike moments, whereas the coefficient method performs efficiently in obtaining a specific order of pseudo-Zernike moments.

\section{References}

[1] P. Nassery and K. Faez, "Signature Pattern Recognition using pseudo-Zernike Moments and A Fuzzy Logic Classifier", IEEE Conference on Image Processing 2, 1996, pp.197-200.

[2] A. Khotanzad and C. Chung, "Hand Written Digit Recognition using Combination of Neural Network Classifiers", IEEE Southwest Symposium on Image Analysis and Interpretation, 1998, pp.168-173.

[3] M. Namazi and K. Faez, "Recognition of multifont Farsi/Arabic characters using a fuzzy neural network", Proceeding of IEEE Region 10 Annual International Conference 2, 1996, pp.918-922.

[4] C.H. Teh and R.T. Chin, "On Image Analysis by the Method of Moments", IEEE Trans. on Pattern Analysis and Machine Intelligence 10(4), 1988, pp.496-513.

[5] S.O.Belkasim, "Pattern Recognition with Moment Invariants - A Comparative Study and New Results", Pattern Recognition 24(12), 1991, pp.1117-1138.

[6] A. Khotanzad, "Invariant image recognition by Zernike moments", IEEE Trans.on Pattern Analysis and Machine Intelligence 12(5), 1990, pp.489-497.

[7] A. Khotanzad and Y.H. Hong, "Rotation invariant image recognition using features selected via a systematic method", Pattern Recognition 23(10), 1990, pp.1089-1101.

[8] R. Mukundan and K.R. Ramakrishnan, "Fast Computation of Legendre and Zernike Moments", Pattern Recognition 28(9), 1995, pp.1433-1442.

[9] X.Y. Jiang, "Simple and fast computation of moments", Pattern Recognition 24(8), 1991, pp.801806.

[10] J.G. Leu, "Computing a shape's moments from its boundary", Pattern Recognition 24(10), 1991, pp.949957.

[11] B.C. Li, "Fast computation of moment invariants", Pattern Recognition 24(8), 1991, pp.807-813.

[12] A. Prata and W.V.T. Rusch, "Algorithm for Computation of Zernike Polynomials Expansion Coefficients", Appl.Opt. 28, 1989, pp.749-754.

[13] S.O. Belkasim, M. Ahmadi and M. Shridhar, "Efficient Algorithm for Fast Computation of Zernike Moments", Circuits and Systems, IEEE 39th Midwest Symposium 3, 1996, pp.1401-1404.

[14] E.C. Kintner, "On the mathematical properties of the Zernike polynomials", Optica Acta 23(8), 1976, pp.679-680.

[15] R. Mukundan and K.R. Ramakrishnan, Moment Functions in Image Analysis- Theory and Applications, World Scientific Publishing, 1998. 
Table 1: Computational Flow of Coefficients of Radial Polynomials

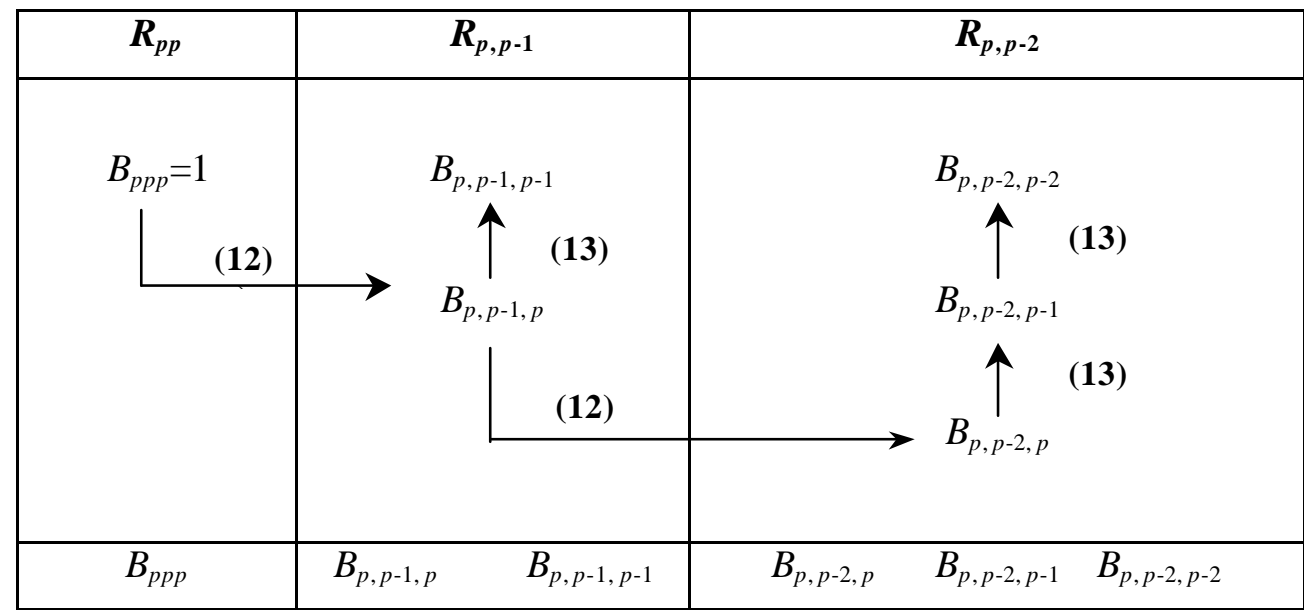
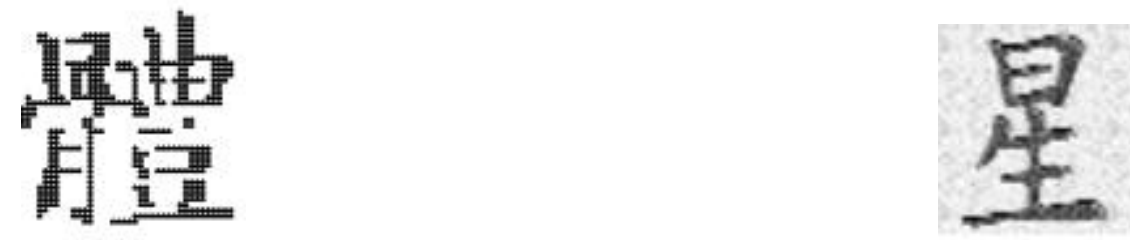

Figure 1 (a) : 50x50-pixel Binary Chinese Character (b): 50x50-pixel Grayscale Chinese Character

Table 2: The CPU EllapsedTime (s) for Computation of Pseudo-Zernike Moments Using Binary Image

\begin{tabular}{|c|c|c|c|c|c|}
\hline $\begin{array}{c}\text { Maximum } \\
\text { Order }\end{array}$ & $\begin{array}{c}\text { Direct } \\
\text { Method }\end{array}$ & $\begin{array}{c}\text { Modified } \\
\text { Belkasim's } \\
\text { Method }\end{array}$ & $\begin{array}{c}\text { Coefficient } \\
\text { Method }\end{array}$ & $\begin{array}{c}\text { Recursive } \\
\text { Method }\end{array}$ & $\begin{array}{c}\text { Two-Stage } \\
\text { Recursive } \\
\text { Method }\end{array}$ \\
\hline NMAX=24 & 8.9 & 1.2 & 1.1 & 0.4 & $\mathbf{0 . 4}$ \\
\hline NMAX=36 & 34.2 & 3.6 & 2.7 & 0.9 & $\mathbf{0 . 7}$ \\
\hline NMAX=48 & 92.6 & 8.3 & 5.8 & 1.4 & $\mathbf{1 . 2}$ \\
\hline
\end{tabular}

Table 3: The CPU EllapsedTime (s) for Computation of Pseudo-Zernike Moments Using Grayscale Image

\begin{tabular}{|c|c|c|c|c|c|}
\hline NMAX=24 & 48.7 & 7.7 & 4.8 & 2.4 & $\mathbf{1 . 9}$ \\
\hline NMAX=36 & 186.3 & 24.7 & 14.6 & 4.8 & $\mathbf{4 . 1}$ \\
\hline NMAX=48 & 495.2 & 56.7 & 32.8 & 8.2 & $\mathbf{7 . 0}$ \\
\hline
\end{tabular}




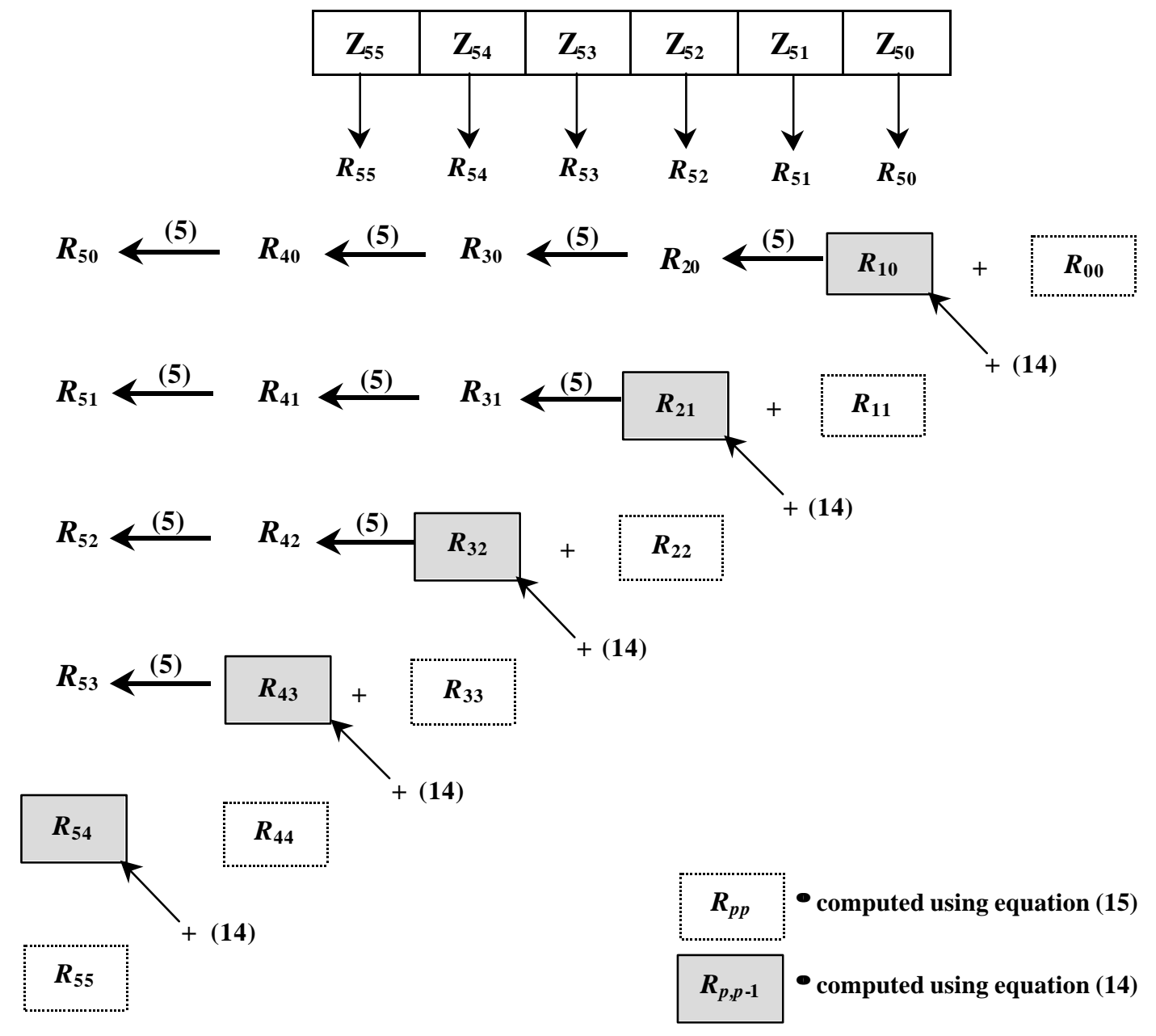

Figure 2: Computation Flow of Two-Stage Recursive Method

Table 4: The CPU EllapsedTime (s) for $48^{\text {th }}$ Order of Pseudo-Zernike Moments

\begin{tabular}{|c|c|c|}
\hline Method & Binary Image & Grayscale Image \\
\hline Coefficient Method & $\mathbf{0 . 3}$ & $\mathbf{1 . 9}$ \\
\hline Two-Stage Recursive Method & 1.2 & 7.0 \\
\hline Recursive Method & 1.4 & 8.2 \\
\hline
\end{tabular}

